\title{
ANTIBIOTIC RESISTANCE PATTERN OF ISOLATES FROM WOUND AND SOFT TISSUE INFECTIONS
}

\author{
Surg Cdr RN MISRA * , Lt Col YOGESH CHANDER ${ }^{+}$, \\ Lt Col NK DEBATA (Retd) ${ }^{\#}$, Brig VC OHRI ${ }^{* *}$
}

\begin{abstract}
Two-hundred and eighty bacterial isolates from wound and soft tissue infections were studied for species identification and antibiotic resistance pattern. Amongst them 122 isolates were from community acquired infection and 158 were from nosocomial infections. The common community acquired pathogens were Staphylococcus aureus $(67.8 \%)$ and Streptococcus pyogenes (10.7\%), whereas Staphylococcus aureus $\mathbf{( 6 0 . 1 \% )}$ and E.Coli $\mathbf{8 . 9 \% )}$ were common in nosocomial infection. Only two anaerobes (Cl perfringens) were isolated. Penicillin resistance was found to be $87 \%$ and $92 \%$ for Staphylococccus aureus in community acquired and noscomial infections respectively. 85\% of Proteus isolates were resistant to ampicillin. There was relatively lower level of resistance by all isolates to cefotaxime. Gentamicin showed higher rate of resistance than netilmicin and amikacin. Resistance of $E$.coli isolates to fluoroquinolones being $79 \%$ for norfloxacin, $81 \%$ for ciprofloxacin and $60 \%$ for ofloxacin. The study showed a higher resistance of methicillin resistant Staphylococcus atreus (MRSA) to other antibiotics. Amikacin and ofloxacin were the best recommended drugs for empirical therapy for all organisms, the susceptibility rate being $80.7 \%$ and $80.4 \%$.
\end{abstract}

MJAFI 2000, $56: 205-208$

KEY WORDS: Antibiotic resistance; Soft tissue infections; Wound infections.

\section{Introduction}

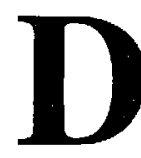
espite the fundamental role of antiseptics and antibiotics in the development of modern surgery, implementation of these practices have reduced but not eliminated post surgical infections. The wound infection depends on a complex interaction between host factors like immunity, nutritional status and age, wound related factors like magnitude of trauma, dead space, devitalization and presence of hematoma and microbial factors like toxins, invasion and resistance to antibiotics. [1]. Different species assume importance in different procedures viz. Staphylococcus aureus and coagulase negative staphylococci in clean $: \cdots$-rical procedures $[2,3]$ and polymicrobial flora e.g. Escherichia coli and bacteroides spp in contaminated procedures like colorectal surgery. The success of prophylaxis in clean surgery correlates directly with suscertibility of bacteria to antibiotics in vitro. This study aims to find out the prevalence of bacterial isolates and their antibiotic resistance pattern so as to facilitate the treating clinicians to select appropriate antibiotics for prophylaxis and treatment and also to develop the bacterial database to study the changes in bacterial behaviour in future.

\section{Material and Methods:}

The study was conducted in the Department of Microbiology, Armed Forces Medical College, and Command Hospital (SC), Pune a tertiary care hospital during the period Jan to Jun 1997.
The pus swabs and body fluid swabs which were received from wound and soft tissue infections from patients attending the outpatient department and those admitted to Command Hospital (SC) were inoculated onto Blood agar, MacConkey agar and Mannite salt agar and incubated at $37^{\circ} \mathrm{C}$ for $18-24$ hours. Pus specimens received in suitable containers i.e. pus swabs in thioglycolate medium or RCM medium or aspirated pus in closed syringe were also inoculated onto blood agar for anaerobic culture. A direct smear for assessment of the cellularity and presence of bacteria was carried out in all cases. The isolates were identified by standard identification methods [4]. Antibiotic sensitivity was carried out by Stokes'disc diffusion method using commercial antibiotic discs or those prepared in our laboratory using pure powder [5]. The standard strains used for this purpose were $S$ aureus NCTC 6571, E Coli NCTC 10418 and P aeruginosa NCTC 10662 [5].

\section{Results}

$S$ aureus constituted the majority of isolates both in the community and hospital infections being $67.2 \%$ and $60.1 \%$ respectively, followed by Str pyogenes in community infections $(10.7 \%)$ and E.coli in nosocomial infections (8.9\%). There were only two (1.3\%) anaerrobic infections due to Clostridium perfringens (Table-1). Majority of $S$ aureus both from community and from hospital were resistant to penicillin being $87 \%$ and $92 \%$ respectively (Table-2). Methicillin resistance among hospital isolates was significantly more (54\%) than the community isolates (43\%). Resistance to Gentamicin amongst the isolate is much more than to netilmicin and amikacin (Table-3). Most of the organisms are sensitive to fluroquinolones particularly to ofloxacin except $E$ coli which were relatively more resistant to those drugs (Table-4). Many $S$ aureus were sensitive to Clindamycin but Proteus spp were $100 \%$ resistant to tetracyclines (Table-5). Resistance pattern of MRSA strains to other antibiotics is given in table-6. Most of the MRSA were resistant to tetracyline, trimethoprim and sul-

"Classified Specialist(Pathology and Microbiology), INHS Asvini, Colaba, Mumbai-400 005, ${ }^{+}$Reader, " Ex-Associate Professor, Department of Microbiology, Armed Forces Medical College, Pune $411040^{* *}$ Commandant, 167 Military Hospital, C/o 56 APO. 
phamethoxazole and a majority to norfloxacin (Table-6). The overall susceptibility of all organisms to newly introduced antibiotics is given in table-7. Susceptibility to amikacin and ofloxacin was more being $80.7 \%$ and $80.4 \%$ respectively. The least susceptibility was shown to gentamicin (52.3\%) and ciprofloxacin $(53.5 \%)$. Two anaerobic isolates were sensitive to most of the antibiotics.

TABLE 1

Isolates from wound and soft tissue infections Jan.-Jun. 1997

\begin{tabular}{lcccc}
\hline Organism & Community Acquired & \multicolumn{2}{c}{ Hospital Acquired } \\
\hline S aureus & $82(67.2)$ & 95 & $(60.1)$ \\
CON Staphylococci & 06 & $(4.9)$ & 08 & $(5.1)$ \\
Strept pyogenes & 13 & $(10.7)$ & 09 & $(5.7)$ \\
E coli & 03 & $(2.5)$ & 14 & $(8.9)$ \\
Proteus spp & 05 & $(4.1)$ & 06 & $(4.0)$ \\
Ps aeruginosa & 09 & $(7.4)$ & 09 & $(5.7)$ \\
Clostridium spp & - & 02 & $(1.3)$ \\
Others & 04 & $(3.3)$ & 15 & $(9.5)$ \\
\hline Total & 122 & 158 & \\
\hline
\end{tabular}

Figures in parenthesis reflect percentage

TABLE 2

Beta-lactam resistance (\%) in major isolates

\begin{tabular}{lcccccc}
\hline & Pen & Amp & Met & Cef & $\mathrm{Ctz}$ & $\mathrm{Cbn}$ \\
\hline S aureus (OPD) 82 & 87 & - & 43 & 29 & - & - \\
$S$ aureus (Hosp) 95 & 92 & - & 54 & 41 & - & - \\
E coli & - & 85 & - & 35 & - & - \\
Proteus spp & - & 63 & - & 11 & - & - \\
Ps aeruginosa & - & - & - & 33 & 40 & 31 \\
\hline
\end{tabular}

Pen = Penicillin; Cef = Cefotaxime; Amp = Ampicillin; $\mathrm{Ctz}=$ Ceftazidime Met $=$ Methicillin; Cbn $=$ Carbenicillin

TABLE 3

Aminoglycoside resistance (\%) in major isolates

\begin{tabular}{lccc}
\hline & Gen & Amk & Net \\
\hline S aureus (OPD) 82 & 42 & - & 18 \\
S aureus (Hosp) 95 & 44 & - & 22 \\
E coli & 65 & 11 & 46 \\
Proteus Spp & 54 & 11 & 10 \\
Ps aeruginosa & 88 & 40 & 63 \\
\hline
\end{tabular}

Gen = Gentamicin, Amk = Amikacin, Net = Netilmicin

TABLE 4

Fluoroquinolones resistance $(\%)$ of major isolates

\begin{tabular}{lccc}
\hline & Nor & Ofl & Cpx \\
\hline S aureus (OPD) 82 & 35 & 19 & 33 \\
S aureus (Hosp) 95 & 45 & 20 & 25 \\
E coli & 79 & 60 & 81 \\
Proteus Spp & 11 & 0 & 22 \\
Ps aeruginosa & 47 & 36 & 53 \\
\hline
\end{tabular}

Nor $=$ Norfloxacin, Ofl $=$ Ofloxacin, $\mathrm{Cpx}=$ Ciprofloxacin
TABLE 5

Other antibacterial resistance (\%) of major isolates

\begin{tabular}{lcccccc}
\hline & Tet & Chl & Sul & Tmp & Cld & Pmx \\
\hline S aureus (OPD) 82 & 56 & 16 & 61 & 60 & 20 & - \\
S aureus (Hosp) 95 & 67 & 36 & 70 & 68 & 23 & - \\
E coli & 94 & 88 & 44 & 86 & - & - \\
Proteus Spp & 100 & 56 & 88 & 63 & - & - \\
Ps aeruginosa & - & - & - & - & - & 00 \\
\hline
\end{tabular}

Tet = Tetracycline; Tmp = Trimethoprim; Chl = Chloromycetin; Cld = Clindamycin; Sul = Sulphamethoxazole; Pmx = Polymyxin B

TABLE 6

Resistance of MRSA to other antibiotics

\begin{tabular}{lccc}
\hline & $\begin{array}{c}\text { MRSA (Hosp) } \\
(\%)\end{array}$ & $\begin{array}{c}\text { MRSA (OPD) } \\
(\%)\end{array}$ & $\begin{array}{c}\text { MRSA(Vellore) } \\
(\%)\end{array}$ \\
\hline Erythromycin & 69.0 & 69.2 & 84.2 \\
Sulphamethoxazole & 92.6 & 81.5 & 97.1 \\
Trimethoprim & 92.3 & 73.0 & 97.1 \\
Tetracycline & 93.3 & 88.5 & 97.9 \\
Chloramphenicol & 59.5 & 61.1 & 72.0 \\
Cefotaxime & 69.2 & 54.2 & - \\
Clindamycin & 41.0 & 21.7 & - \\
Gentamicin & 66.6 & 71.4 & 85.5 \\
Netilmicin & 30.7 & 64.7 & 12.0 \\
Norfloxacin & 80.0 & 66.6 & 90.0 \\
Ofloxacin & 33.3 & 40.0 & 53.0 \\
Ciprofloxacin & 40.0 & - & 92.2 \\
Vancomycin & 2.4 & 00 & 00 \\
Amikacin & - & - & 45.2 \\
\hline
\end{tabular}

TABLE 7

Susceptibility of all organisms to newer antibiotics

\begin{tabular}{lc}
\hline Antibiotic & Susceptibility (\%) \\
\hline Cefotaxime & 66.5 \\
Gentamicin & 52.3 \\
Netilmicin & 75.3 \\
Amikacin & 80.7 \\
Ciprofloxacin & 53.5 \\
Norfloxacin & 57.6 \\
Ofloxacin & 80.4 \\
\hline
\end{tabular}

\section{Discussion}

$S$ aureus was the commonest isolate from the community as well as nosocomial infections of wound and soft tissue. This could be due to the patient's endogenous flora, where the skin and the appendages are usually colonised by this organism [2]. However, rarely a particular surgeon or nurse is responsible for a cluster of staphylococcal infection. Strokes et al [6] found wound infection due to $S$ aureus $17 \%$, enterococci $13 \%$, coagulase negative staphylococci $12 \%$ and $E$ coli $10 \%$ [6]. However, selected populations such as diabetic individuals and patients on haemodialysis 
may be colonised with $S$ aureus at a frequency in excess of 50\% [7]. Many of our patients suffered from diabetes mellitus and/or peripheral vascular disease. More than one isolate was observed in such patients.

After the discovery of $\beta$-lactamase in 1944 , its production among the staphylococci is steadily increasing. Today it is produced in approximately $90 \%$ of staphylococcal isolates from community and hospital which are resistant to penicillin [8]. In this study penicillin resistance was $87 \%$ for community isolates and 92\% for hospital isolates. Due to the plasmid and chromosomally mediated $\beta$-lactamase production among gram negative organisms, they were resistant to ampicillin and other $\beta$-lactams. In this study ampicillin resistance was seen among $88 \% E$ coli and 65\% Proteus spp. Resistance to cefotaxime, a third generation cephalosporin was relatively low (Table-2).

Gentamicin resistance was higher among isolates as it was introduced earlier than netilmicin and amikacin (Table-3). Many strains with plasmid encoded extended spectrum $\beta$-lactamase were also aminoglycoside resistant. So large out-breaks of infection with such strains may result in an increase in aminoglycoside resistance rates [9]. Gentamicin resistance in our centre during 1996-97 was more than that found in an UK multicentric study where only $0.6 \% \mathrm{E}$ coli, $6 \%$ of $K$ pneumoniae, and $13 \%$ of $K$ oxytoca were resistant to this aminoglycoside [10]. This could be due to more chronic nature of the cases in our study and the organisms with constant exposure to gentamicin in the hospital environment.

Resistance to fluoroquinolones occurs in organisms with high initial MIC including Ps aeruginosa, MRSA (with $80 \%$ resistance), coagulase negative staphylococci and A-inetobacter spp. Emergence of resistance during treatment with quinolones is most likely to occur when large number of organisms present at the site of infection are exposed to subinhibitory concentration of antibiotics either because of inadequate dosage or because of impaired antibiotic penetration e.g. prolonged or repeated chronic infection, impaired vascularity, osteomyelitis or presence of foreign bodies. Strategy to reduce resistance is to prescribe two to three drugs for the first 2-5 days followed by quinolones after reduction of the bacterial load [10]. In our series the resistance of $E$ coli was $79 \%$ for norfloxacin, $60 \%$ for ofloxacin and $81 \%$ for ciprofloxacin but Proteus spp showed complete susceptibility to ofloxacin.

Among the $E$ coli and Proteus spp, 94\% and $100 \%$ were resistant to tetracyline respectively. $S$ aureus is relatively less resistant to chloramphenicol, $16 \%$ and $36 \%$ of community and hospital isolate respectively, possibly due to under-exposure to it. Clindamycin is still quite effective for $S$ aureus, only $20 \%$ of isolates being resistant to it.

The MRSA strains show higher resistance to other antibiotics as well. They show $92.6 \%$ resistance to sulphamethoxazole, $92.3 \%$ to trimethoprim, $93.3 \%$ to tetracycline, and $80 \%$ to norfloxacin. The community isolates of MRSA show $81.5 \%$ resistance to sulphamethoxazole, $88 \%$ to trimethoprim. In another Indian study [12] $24 \%$ of staphylococci were MRSA which showed $97 \%$ resistance to trimethoprim, $85.5 \%$ to gentamicin, $45 \%$ to amikacin, $90 \%$ to norfloxacin and ciprofloxacin and 55\% to ofloxacin. They recommend netilmicin as alternative drug for MRSA strains (Table-6), but we recommend vancomycin and ofloxacin for hospital isolates. Clindamycin and ofloxacin can be administered to community isolates of MRSA.

The characteristic of MRSA is that they produce altered penicillin binding proteins (PBP) $2 \mathrm{a}$ or 2 which are chromosomally transmitted. The other characteristics of MRSA include penicillinase production and a full armamentarium of pathogenic factors that inlude protein A, coagulase and DNAse. Numerous in vitro studies have shown MRSA to be fully virulent and showed intraphagocytic killing potential as well resistant to a multitude of antibiotics [13-16].

The percentage susceptibility of all organisms to various newer antibiotics shows that highest number of organisms are susceptible to amikacin $(80.7 \%)$, followed by ofloxacin $(80.4 \%$ ) and netilmicin (75.3\%) (Table-7). This will help the treating physicians to decide on empirical antibiotic therapy until the sensitivity report is available.

\section{REFERENCES}

1. Kemodle DS, Kaiser AB. Post operative infections and antimicrobial prophylaxis. In: Mandell GL, Bennett JE, Dolin R, eds. Principles and Practice of Infectious Diseases. 4th ed. New York, Churchill Livingstone 1995:2742-56.

2. Ketcham AS, Lieberman JE, West JI. Antibiotic prophylaxis and cancer surgery and its value in staphylococcal carrier patients. Surg Gynaecol Obstet 1963;117:1-6.

3. Weinstein HJ. The relation between the nasal staphylococcal carrier state and the incidence of post operative complications. N Engl J Med 1959;260:1303-7.

4. Collee JG, Fraser AG, Marmion BP, Simmons A. Mackie and McCartney Practical Medical Microbiology. 14th ed. New York, Churchill Livingstone, 1996:95-150.

5. A guide to sensitivity testing: Report of working party on antibiotic sensitivity testing of the Brisish Society for Antimicrobial Chemotherapy. J Antimicrob Chemotherap 1991;27: Supplement D: $1-50$. 
6. Stokes EJ, Ridgway GL, Wren MWD. Clinical Microbiology. 7th ed. Boston, Edward Amold 1993:251-2.

7. Schaberg DR, Culver DH, Gaynes RP. Major trends in the microbial aetiology of nosocomial infections. Am J Med 1991;91(Suppl 3B): 72-5.

8. Kirmani N, Tuazon CU, Murray HW. Staphylococcus aureus Arch Intern Med 1978;138:1657-9.

9. R Suderland. $\beta$-lactams: penicillins. In: $O^{\prime}$ Grady F, Finch RG, Lambert HP, Greenwood D, eds. Antibiotic and Chemotherapy. 7th ed. New York, Churchill Livingstone 1997:256305.

10. Fisher GM, Kelsey MC, Corky EM. An investigation of the spread of gentamicin resistance in a district general hospital. $\mathrm{J}$ Med Microbiol 1986;22:69-77.

11. Brown EM, Reeves DS. Quinolones. In: O'Grady F, Lambert HP, Finch RG, Greenwood D, eds. Antibiotic and Chemotherapy. 7th edn. New York, Churchill Livingstone 1997:419-52.
12. Pulimood TB, Lalitha MK, Jesudasan MV, Pandian R, Selwyn J, John TJ. The spectrum of antimicrobial resistance among Methicillin Resistant Staphylococcus aureus in a tertiary care centre in India. Indian J Med Res 1996;103:212-5.

13. Hartman BM, Tomasz A. Low affinity penicillin binding protein associated with $\beta$-lactam resistance in Staphylococcus aureus. J Bacteriol 1984; 158: 513-7.

14. Peacock JE, Moorman DR, Wenzel RP. Methicillin resistant Staphylococcus aureus: Microbiologic characteristics, antimicrobial susceptibilities and assessment of virulence of an epidemic strain. J Infect Dis 1981:144:575-7.

15. Kinsman O, Naidoo J, Noble WC. Some effects of plasmid coding for antibiotic resistance on the virulence of Staphylococcus aureus. Pathology 1985;66:325-30.

16. Shalit I, Berger SA, Gorea A. Widespread quinolone resistance among methicillin resistance Staphylococcus aureus isolates in a general hospital. Antimicrob Agents Chemother 1989;33:595-7. 